\title{
A Lethal Sequelae of Spinal Infection Complicating Surgery and Radiotherapy for Head and Neck Cancer
}

\author{
Jason Pui Yin Cheung ${ }^{1}$, Kin Cheung Mak ${ }^{1}$, Helen Hoi Lun Tsang ${ }^{2}$, Keith Dip Kei Luk ${ }^{1}$ \\ ${ }^{1}$ Department of Orthopaedics and Traumatology, The University of Hong Kong, Pokfulam, Hong Kong SAR, China \\ ${ }^{2}$ Department of Medicine, The University of Hong Kong, Pokfulam, Hong Kong SAR, China
}

Patients who have undergone neck dissection and radiotherapy are at risk of cervical spine infections. Furthermore, previous radiotherapy and cervical spine infections can lead to fistula formation to the subarachnoid space and intracranial infection. This report discusses the serious consequences of a missed cervical spine infection including cerebrospinal fluid fistula formation and persistent central nervous system infection, and serves as a reminder to clinicians of the possible association between cervical spine infections and prior head and neck surgery and radiotherapy. In all such cases, the posterior pharyngeal wall should be inspected during followup. Despite the appearance of an intracranial infection, the cervical spine should be investigated, especially if the response to appropriate antibiotics is suboptimal.

Keywords: Spondylodiscitis; Radiotherapy; Cervical spine; Cerebrospinal fluid fistula

\section{Introduction}

We report the case of a patient who had undergone laryngopharyngeal surgeries and radiotherapy presenting with spondylodiscitis, cerebrospinal fluid (CSF) fistula, and pneumocephalus. Patients with a history of neck dissection or radiotherapy may be at risk of developing cervical spine infections, and thus, the integrity of the posterior pharyngeal wall should be assessed in all at-risk patients. Furthermore, fistulation to the spinal canal can be caused by the infective process and lead to morbid sequelae of meningitis and pneumocephalus. In patients with persistent mucosal defects, flap coverage is recommended [1]. This case report demonstrates that any patient with an intracranial infection and a history of prior laryngopharyngeal surgeries and suboptimal response to appropriate antibiotic therapy should undergo a thorough investigation of the cervical spine for potential primary infective focus.

\section{Case Report}

A 66-year-old man presented with fever, headache, generalized tonic clonic seizure and neck stiffness three months after partial pharyngectomy for squamous cell carcinomain-situ at the posterior pharyngeal wall. He had history of laryngeal cancer, which was treated by micro-laryngectomy and adjuvant radiotherapy, six years prior to this presentation, and of esophageal cancer with esophagectomy, which was treated by selective neck dissection and radiotherapy, some four years previously. An endoscopic

Received Sep 21, 2014; Accepted Oct 16, 2014

Corresponding author: Keith Dip Kei Luk

Tam Sai Kit Endowed Chair in Spine Surgery, Department of Orthopaedics and Traumatology, The University of Hong Kong, Professorial Block, 5th Floor, 102 Pokfulam Road, Pokfulam, Hong Kong SAR, China

Tel: +852-2255-4254, Fax: +852-2817-4392, E-mail: hrmoldk@hku.hk 
evaluation performed after his last operation revealed no evidence of local recurrence.

As his symptoms were suggestive of meningitis, brain magnetic resonance imaging (MRI) was performed, and showed generalized leptomeningeal enhancement. Analysis of CSF obtained by lumbar puncture revealed an increased white cell count (WCC) and protein level and a decreased glucose level, but microbiological studies were negative. He was treated for meningitis and was given intravenous ceftriaxone and acyclovir, and oral dexamethasone to reduce cerebral edema. Repeat MRI performed two weeks later showed decreased leptomeningeal enhancement and repeat lumbar puncture revealed normalized WCC and biochemical markers in CSF.

Four weeks after the complete cessation of antibiotics, he developed dizziness and nystagmus in right horizontal gaze. Brain computed tomography (CT) (Fig. 1) showed a small right caudate and cerebellar infarct and loculated air densities at the interhemispheric fissure, left Sylvian fissure, interpeduncular cistern, left ambient cistern, and pericallosal region. In view of the new clinical and imag-

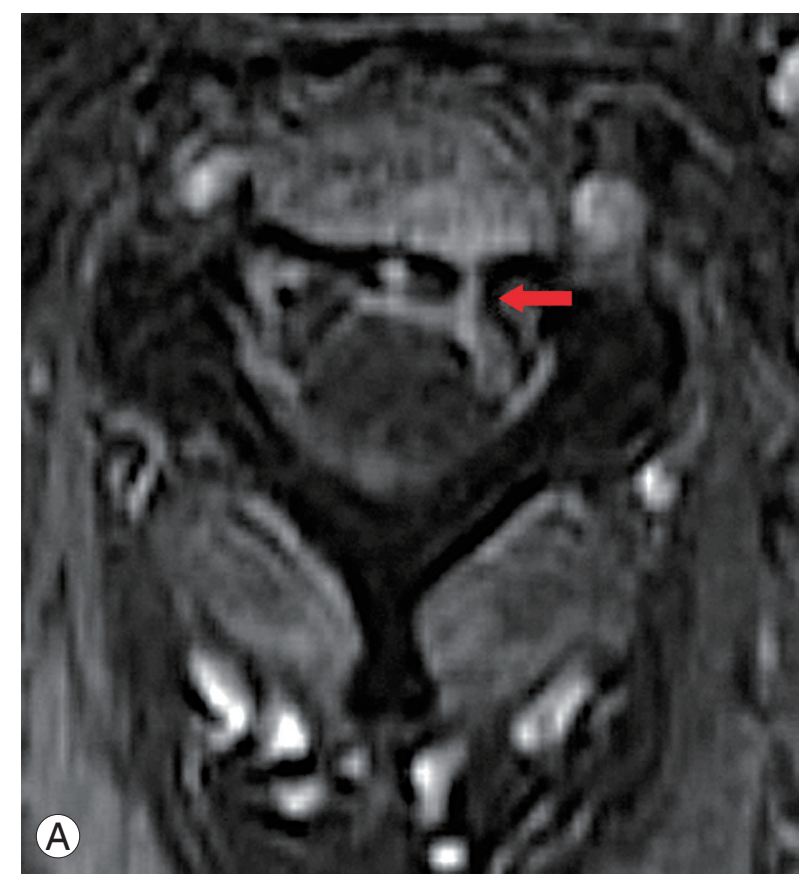

Fig. 2. (A) Axial magnetic resonance imaging (MRI) T1 contrast image showing the C3-C4 cerebrospinal fluid fistula (red arrow) to the subarachnoid space. (B) Sagittal MRI T1 contrast image showing the C3-C4 spondylodiscitis (white arrow). ing findings and his history of head and neck cancers, neck MRI was performed to investigate the source of the infection and pneumocephalus. MRI (Fig. 2) revealed

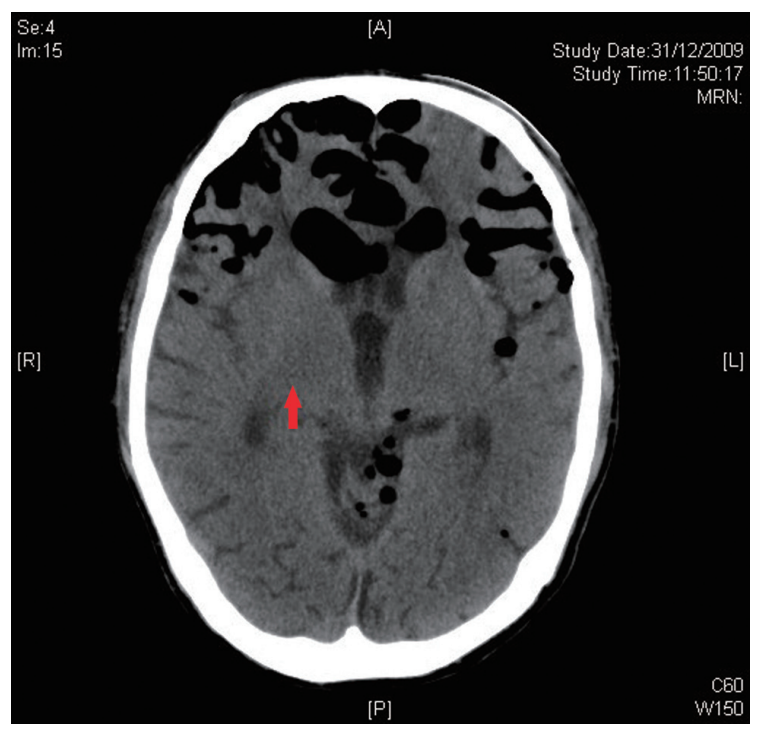

Fig. 1. Computed tomography brain showing right caudate infarct (red arrow) and gross pneumocephalus.

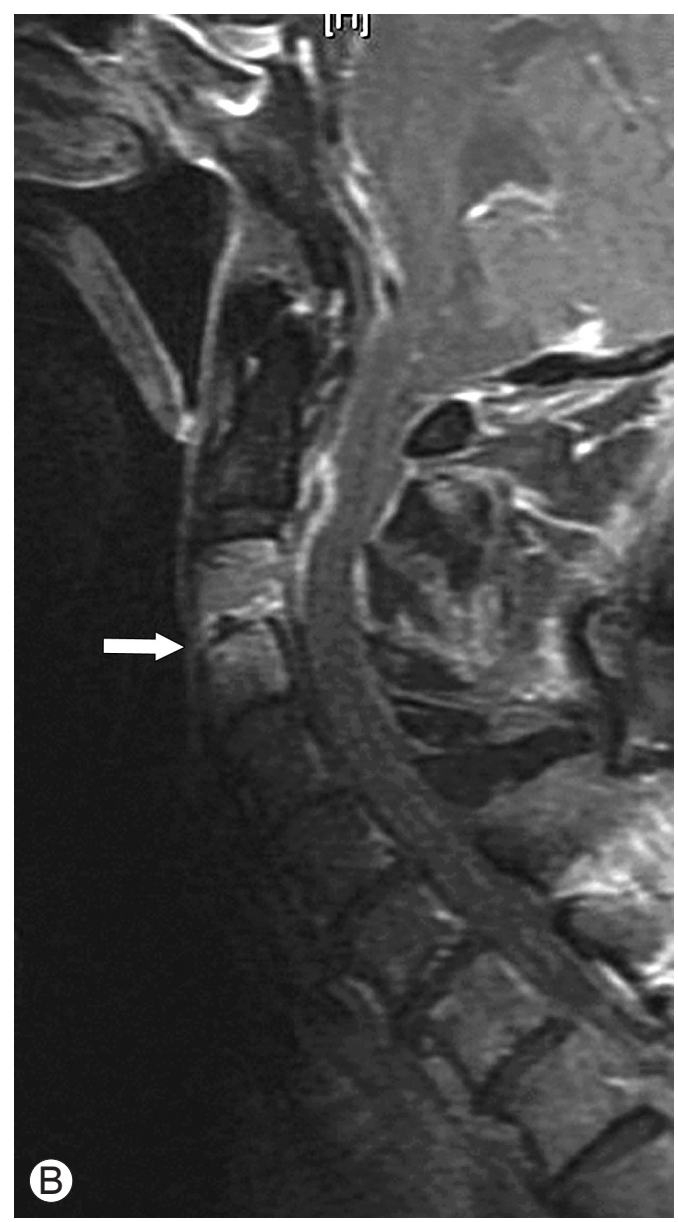


C3-C4 spondylodiscitis and a CSF fistula to the subarachnoid space, and CT myelography (Fig. 3) demonstrated the site of CSF leakage through the dura at the level of the C3-C4 disc. Vancomycin, cefepime, and metronidazole were started after consultation with a microbiologist.

Unfortunately, the patient failed to respond and became tetraplegic and required mechanical ventilation shortly afterwards and subsequently suffered from repeated chest infections. Due to his poor general condition and the high surgical risk, surgical debridement and excision of the fistula were abandoned. Intravenous antibiotics were continued but he succumbed due to uncontrolled sepsis at 2 months after the diagnosis was made.

\section{Discussion}

Surgical neck dissection is important in head and neck surgeries to achieve adequate clearance of tumor tissues. However, this procedure undoubtedly damages peripheral lymphoid tissues and leads to deficiencies in regional

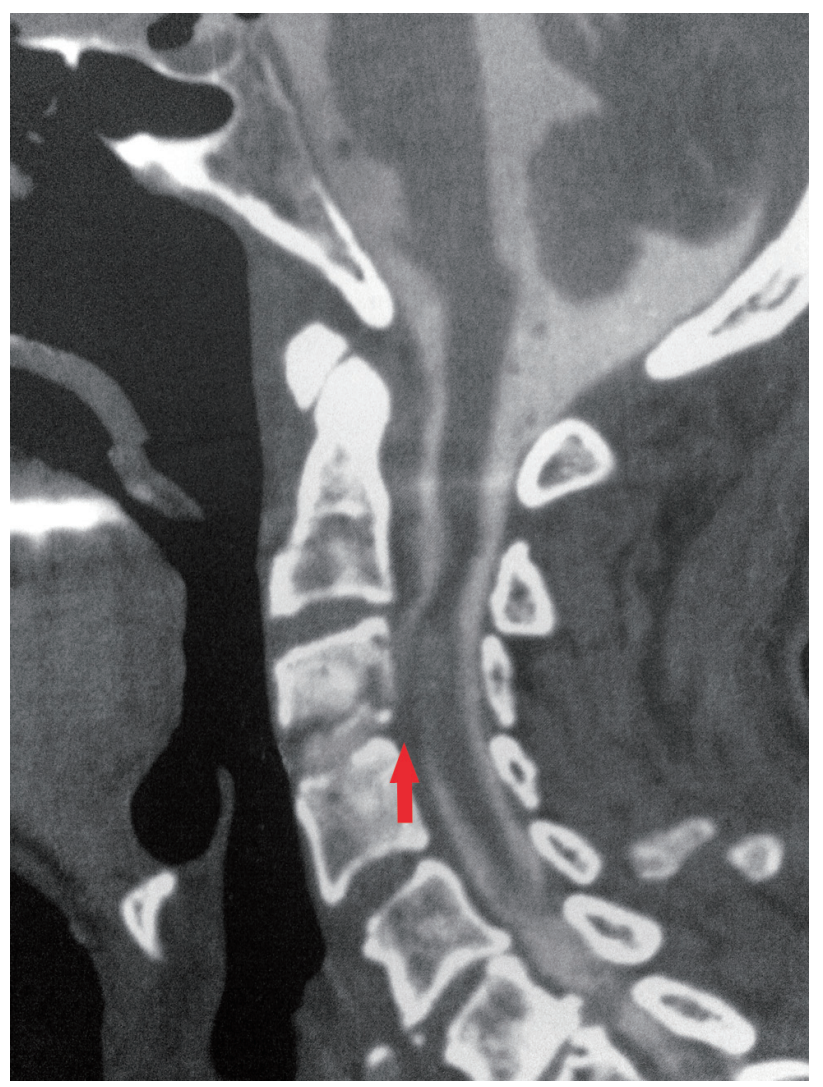

Fig. 3. Computed tomography myelogram showing C3-C4 spondylodiscitis, C3 vertebral body destruction, and the connecting cerebrospinal fluid fistula (red arrow) resistance to infection $[1,2]$. Further damage to the lymphoid barrier and posterior pharyngeal mucosal defects can be attributed to the radiotherapy given for local tumor control [2], and infective organisms from the oropharyngeal cavity can gain access to the cervical spine via these mucosal defects [3]. In addition, radiotherapy can enhance the permeability of the blood-brain barrier, and thereby, increase the risk of central nervous system (CNS) infections [4].

Similar problems occur in patients suffering from nasopharyngeal carcinoma treated by surgery and radiotherapy. In these at-risk patients, regular assessment of the posterior pharyngeal wall should be performed at every follow-up consultation as infections may occur even at 17 years after radiotherapy [1]. Transoral examination using a torch and tongue depressor provides a simple and economical means of assessing pharyngeal mucosa. Patients with pharyngeal wall defects should receive flap coverage to prevent further access of the cervical spine by oropharyngeal microorganisms. The commonly used pedicle flaps include the latissimus dorsi, paraspinal muscle, and trapezius flaps [5].

As illustrated by our case, multiple neck surgeries and radiotherapy had weakened the lymphoid barrier in the head and neck region, and contributed to the development of spondylodiscitis via the direct spread of bacteria from the oropharyngeal region. Compounded by delayed diagnosis of the fistula, this infective process spread posteriorly through the disc to reach the epidural space in our patient and led to uncontrolled intracranial infection. The CSF fistula was likely to have occurred secondary to spondylodiscitis as there was no previous neurosurgical procedure.

CSF fistulas should be treated early as persistent CSF leakage prevents proper healing [6]. The principles of treatment involve infection control, improving overall immunity, direct surgical repair of the fistula, reinforcement of the repair with a muscle flap, and obliteration of any dead space [7]. Conservative treatment with antibiotics usually fails [8] and surgical treatment including external lumbar drainage, direct dural repair, duraplasty, and in some cases coverage with fat, fascia or synthetic grafts is required to prevent suture failure or fistula recurrence [7,9-12].

The delay in establishing the link between the CNS infection and previous neck surgery and radiotherapy was the main management downfall in our case. The causative 
pathology was only discovered after the patient experienced significant neurological deterioration and exhibited poor response to antibiotic treatment. Any salvage surgery at this point would have already been too late. The initial presentations of headache, convulsions and neck stiffness suggested a diagnosis of meningitis. To a more cautious clinician, a history of multiple neck operations and radiotherapy would have triggered further investigations for cervical spine pathologies. With more vigilance, the culprit could have been identified earlier and the window of opportunity for surgical management would not have been missed. Patient condition permitting, the proper management would have been thorough debridement, fistula excision and soft tissue coverage of the defect to prevent recurrence. In our case, despite potent antibiotics, the infection was not controlled because the fistula was not obliterated.

\section{Conflict of Interest}

No potential conflict of interest relevant to this article was reported.

\section{References}

1. Cheung JP, Wei WI, Luk KD. Cervical spine complications after treatment of nasopharyngeal carcinoma. Eur Spine J 2013;22:584-92.

2. King AD, Griffith JF, Abrigo JM, et al. Osteoradionecrosis of the upper cervical spine: MR imaging following radiotherapy for nasopharyngeal carcinoma. Eur J Radiol 2010;73:629-35.

3. Lim AA, Karakla DW, Watkins DV. Osteoradionecrosis of the cervical vertebrae and occipital bone: a case report and brief review of the literature. Am J Otolaryngol 1999;20:408-11.

4. Fernandez-Ruiz M, Lopez-Medrano F, Garcia-Mon- tero M, Hornedo-Muguiro J, Aguado JM. Intramedullary cervical spinal cord abscess by viridans group Streptococcus secondary to infective endocarditis and facilitated by previous local radiotherapy. Intern Med 2009;48:61-4.

5. Kouyoumdjian P, Gille O, Aurouer N, Soderlund C, Vital JM. Cervical vertebral osteoradionecrosis: surgical management, complications and flap coverage: a case report and brief review of the literature. Eur Spine J 2009;18 Suppl 2:258-64.

6. Waisman M, Schweppe Y. Postoperative cerebrospinal fluid leakage after lumbar spine operations. Conservative treatment. Spine (Phila Pa 1976) 1991; 16:52-3.

7. Hyun SJ, Rhim SC, Ra YS. Repair of a cerebrospinal fluid fistula using a muscle pedicle flap: technical case report. Neurosurgery 2009;65:E1214-5.

8. Matsumoto M, Watanabe K, Ishii K, et al. Complicated surgical resection of malignant tumors in the upper cervical spine after failed ion-beam radiation therapy. Spine (Phila Pa 1976) 2010;35:E505-9.

9. Fountas KN, Kapsalaki EZ, Johnston KW. Cerebrospinal fluid fistula secondary to dural tear in anterior cervical discectomy and fusion: case report. Spine (Phila Pa 1976) 2005;30:E277-80.

10. Ozgen S, Boran BO, Elmaci I, Ture U, Pamir MN. Treatment of the subarachnoid-pleural fistula: case report. Neurosurg Focus 2000;9:ecp1.

11. Raffa SJ, Benglis DM, Levi AD. Treatment of a persistent iatrogenic cerebrospinal fluid-pleural fistula with a cadaveric dural-pleural graft. Spine J 2009;9:e25-9.

12. Robertson JT, Soble-Smith J, Powers N, Nelson PA. Prevention of cerebrospinal fistulae and reduction of epidural scar with new surgical hemostat device in a porcine laminectomy model. Spine (Phila Pa 1976) 2003;28:2298-303. 